\title{
Celecoxib-mediated activation of endoplasmic reticulum stress induces de novo ceramide biosynthesis and apoptosis in hepatoma HepG2 cells
}

\author{
Hyo Jin Maeng ${ }^{1, \#}$, Jae-Hwi Song ${ }^{1, \#}$, Goon-Tae Kim ${ }^{1}$, Yoo-Jeong Song ${ }^{1}$, Kangpa Lee ${ }^{2}$, Jae-Young Kim ${ }^{1}$ E Tae-Sik Park, \\ ${ }^{1}$ Department of Life Science, Gachon University, Seongnam 13120, ${ }^{2}$ Department of Physiology, Konkuk University School of Medicine, \\ Seoul 05030, Korea
}

\begin{abstract}
Ceramides are the major sphingolipid metabolites involved in cell survival and apoptosis. When HepG2 hepatoma cells were treated with celecoxib, the expression of the genes in de novo sphingolipid biosynthesis and sphingomyelinase pathway was upregulated and cellular ceramide was elevated. In addition, celecoxib induced endoplasmic reticulum (ER) stress in a time-dependent manner. SPTLC2, a subunit of serine palmitoyltransferase, was overexpressed by adenovirus. Adenoviral overexpression of SPTLC2 (AdSPTLC2) decreased cell viability of HEK293 and HepG2 cells. In addition, AdSPTLC2 induced apoptosis via the caspase-dependent apoptotic pathway and elevated cellular ceramide, sphingoid bases, and dihydroceramide. However, overexpression of SPTLC2 did not induce ER stress. Collectively, celecoxib activates de novo sphingolipid biosynthesis and the combined effects of elevated ceramide and transcriptional activation of ER stress induce apoptosis. However, activation of de novo sphingolipid biosynthesis does not activate ER stress in hepatoma cells and is distinct from the celecoxib-mediated activation of ER stress. [BMB Reports 2017; 50(3): 144-149]
\end{abstract}

\section{INTRODUCTION}

Ceramide is a component of the plasma membrane and a bioactive signaling molecule involved in regulating cellular events including cell cycle arrest, apoptosis, senescence, and stress responses (1). Ceramide can be produced from the de novo pathway and the sphingomyelinase pathway via sphingomyelinase (2). Formation of ceramide is induced by

${ }^{*}$ Corresponding author. Tel: +82-31-750-8824; Fax: +82-31-7508573; E-mail: tspark@gachon.ac.kr

${ }^{\#}$ These authors contributed equally to this work.

https://doi.org/10.5483/BMBRep.2017.50.3.197

Received 30 November 2016, Revised 21 December 2016, Accepted 6 February 2017

Keywords: Apoptosis, Celecoxib, Ceramide, ER stress, Sphingolipid tumor necrosis factor- $\alpha$ (TNF- $\alpha$ ), Fas ligand, oxidative stress, heat stress, and chemotherapeutics (3-5). The balance of ceramide and sphingosine-1-phosphate (S1P), a sphingolipid metabolite that induces cell proliferation, is called "sphingolipid rheostat" and is known to determine cell fate (6).

Chemotherapeutic agents induce ceramide synthesis via activation of dihydroceramide desaturase or activation of de novo pathways $(7,8)$. Celecoxib, an inhibitor of cyclooxygenase 2 (COX2), induces cell cycle arrest and apoptosis in various cancer cells including colon, lung and cervical cancers (9-11). In addition to its inhibitory effect on COX2, celecoxib activates modulatory effects on sphingolipid biosynthesis and elevates cellular ceramide (12). Celecoxib-mediated ceramide elevation activates signaling proteins such as cathepsin D, protein kinase $\zeta$, and protein phosphatase- 1 and contributes to the progression of apoptotic events (13).

The endoplasmic reticulum (ER) is the organelle responsible for protein folding, lipid synthesis, and protein maturation. ER stress is induced by the accumulation of unfolded proteins in the ER lumen, leading to activation of the unfolded protein response and the transcriptional upregulation of chaperones (14). Various studies have demonstrated that ER stress leads to growth arrest and apoptosis in carcinoma cells $(15,16)$. Fumonisin B1, a ceramide synthase (CerS) inhibitor, inhibits ceramideinduced ER stress and alteration of ceramide synthase 6 (CerS6) up-regulates the activating transcription factor 6 (ATF6)-CCAAT-enhancer-binding protein homologous protein (CHOP) pathway and apoptosis in human head and neck squamous cell carcinomas $(17,18)$. However, activation of ER stress and sphingolipid synthesis by celecoxib in hepatoma cells remains unexplored.

The present studies were undertaken to determine whether celecoxib is a transcriptional activator of de novo sphingolipid biosynthesis and induces ceramide-mediated apoptosis in hepatoma cells. Furthermore, we examined whether the activated de novo sphingolipid biosynthetic pathway mediates elevation of cellular ceramide regulates ER stress. Collectively, the results identified the apoptotic effects of celecoxib on hepatoma cells via the activation of ceramide synthesis and ER stress.

ISSN: 1976-670X (electronic edition)

Copyright (C) 2017 by the The Korean Society for Biochemistry and Molecular Biology

(c) This is an open-access article distributed under the terms of the Creative Commons Attribution Non-Commercial License (http://creativecommons.org/licenses/by-nc/4.0) which permits unrestricted non-commercial use, distribution, and reproduction in any medium, provided the original work is properly cited. 


\section{RESULTS}

\section{Celecoxib transcriptionally upregulates de novo sphingoliopid biosynthetic genes and elevates sphingolipid metabolites}

Celecoxib is implicated in the elevation of cellular sphingolipids (12). Therefore, we investigated whether celecoxib transcriptionally upregulates SPTLC1 and SPTLC2. SPTLC1 and SPTLC2 are the two major subunits of serine palmitoyltransferase (SPT), which is the first and rate-limiting step of the sphingolipid biosynthetic pathway. We treated HepG2 cells with celecoxib at various time points. Eighty $\mu \mathrm{M}$ of celecoxib was chosen for this study because of its maximum inducing effect (data not shown). The expression of SPTLC1 and SPTLC2 mRNA and proteins was upregulated by celecoxib and SPT activity increased two-fold (Fig. 1A-C). To determine whether celecoxib alters the transcription of other sphingolipid biosynthetic genes, we measured the expression of genes in the de novo and sphingomyelinase pathways. We found that all ceramide synthase 1, 2, 3, 5, 6, alkaline ceramidases 2-3, acid ceramidase, and neutral ceramidase were upregulated in a time-dependent manner (Fig. 1D). However, the expression of dihydroceramide desaturase, sphingosine kinase 2, and ceramide synthase 4 was not altered. In addition, sphingomyelinases were also upregulated in a time-dependent manner (Fig. 1D). These results suggest that celecoxib-mediated ceramide elevation was due to the transcriptional activation of both the

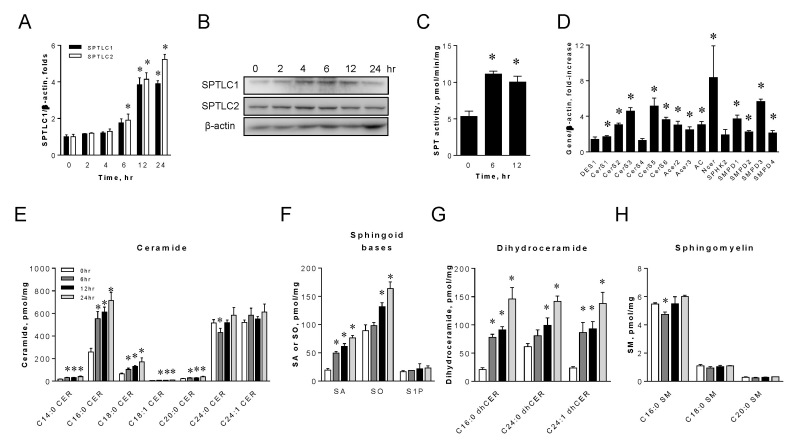

Fig. 1. Celecoxib upregulates the expression of sphingolipid biosynthetic genes and elevates cellular sphingolipid levels. HepG2 cells were treated with $80 \mu \mathrm{M}$ celecoxib and were harvested at various time points. Expression levels of SPTLC1 and SPTLC2 mRNA (A) and proteins (B) were measured by quantitative real-time PCR and immunoblotting respectively. SPT enzyme activity was measured from the cell lysates at indicated times post-celecoxib treatment $(\mathrm{C})$. Expression of sphingolipid biosynthetic genes was measured as the relative fold-increase, compared to no-treatment controls (D). Sphingolipids from HepG2 cells treated with celecoxib at indicated times were analyzed by LC/MS/MS as described in Materials and Methods. Total ceramides (E), sphinganine (SA), sphingosine (SO), and sphingosine 1-phosphate (S1P) (F), dihydroceramide (dhCer) (G), and sphingomyelin (SM) (H) were quantified. Data are presented as the mean \pm SEM. $n=3$. $* P<0.05$. Amount of mRNA was normalized by $\beta$-actin. de novo and sphingomyelinase pathways.

Transcriptional activation of ceramide biosynthesis by celecoxib implies that sphingolipids would be elevated in HepG2 hepatoma cells. To assess this, we measured sphingolipids in HepG2 cells by LC/MS/MS after treatment with celecoxib at various time points. Total ceramide levels were elevated by celecoxib. The levels of long chain ceramides, including C16:0 and C18:0 ceramide, were elevated in a time-dependent manner, but the levels of very long chain ceramides, including C24:0 and C24:1 ceramide, were not changed (Fig. 1E). Sphinganine and sphingosine were elevated but S1P was not altered (Fig. 1F). In addition, all dihydroceramides were increased in a time-dependent manner (Fig. 1G). In contrast, sphingomyelin levels were not altered by celecoxib (Fig. 1H). These findings indicate that celecoxib-mediated induction of sphingolipid biosynthetic genes leads to the activation of de novo sphingolipid biosynthesis and the elevation of sphingolipid levels. Increased levels of cytotoxic long chain ceramides implicate celecoxib in the production of apoptotic long chain ceramide and the initiation of apoptotic events.

\section{Celecoxib activates ER stress in HepG2 hepatoma cells}

Next, we tested whether celecoxib activates ER stress in HepG2 cells. When we treated cells with celecoxib, mRNA levels of ATF4, ATF6, spliced XBP1 (sXBP1), GRP78, and $\mathrm{CHOP}$ were upregulated in a time-dependent manner (Fig. $2 A-E)$. We also found that celecoxib increased the protein levels of ATF4, ATF6, sXBP-1, unspliced XBP1 (uXBP1), and CHOP until 6 hours of post-treatment (Fig. 2F). After 12 hours, celecoxib-induced expression of these proteins disappeared.

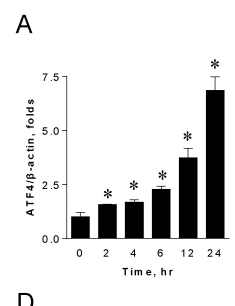

B B
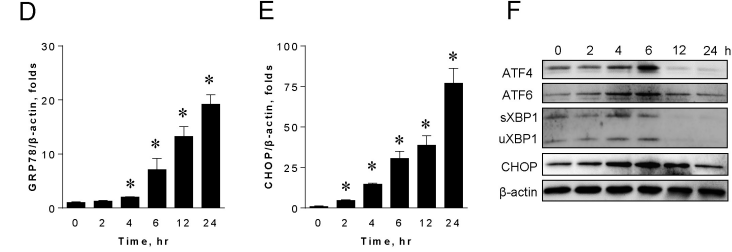

Fig. 2. Celecoxib upregulates ER stress in HepG2 hepatoma cells HepG2 cells were harvested at indicated times after celecoxib treatment and the mRNA expression of ER stress markers, including ATF4 (A), ATF6 (B), spliced XBP1 (sXBP1) (C), GRP78 (D), and CHOP (E), was measured by quantitative real-time PCR. Amount of mRNA was normalized by $\beta$-actin. Data are presented as the mean \pm SEM. $n=3 .{ }^{*} P<0.05$. Whole-cell lysates were subjected to immunoblotting analyses of ATF4, ATF6, XBP-1, and $\mathrm{CHOP}(\mathrm{F})$. $\beta$-actin was used to normalize the mRNA amount. 
This was probably due to the combined cytotoxic effects of celecoxib and ceramide-mediated apoptotic events. Interestingly, ATF6 and CHOP remained elevated until 12 hours of posttreatment (Fig. 2F). These results suggest that celecoxib is responsible for the activation of ER stress in HepG2 hepatoma cells.

\section{Adenoviral expression of SPTLC2 is cytotoxic and elevates cellular ceramide}

The finding that celecoxib activates de novo sphingolipid biosynthesis and ER stress prompted us to distinguish celecoxibdependent events from ceramide effects. For this, we constructed adenoviruses containing SPTLC1 (AdSPTLC1) and SPTLC2 (AdSPTLC2). These are the major subunits of SPT, the first and rate-limiting step in sphingolipid biosynthesis. During adenovirus production in HEK293 cells, SPTLC1 did not affect the adenovirus yield and followed the regular processes of adenovirus production. However, SPTLC2 causes cell death and low adenovirus yield (Fig. 3A). When we treated HepG2 cells with these adenoviruses, SPTLC1 or SPTLC2 protein levels were significantly elevated (Fig. 3B). Adenoviral infection did not affect 24 hour cell viability. AdSPTLC2 infection reduced cell viability by $51 \%$ within 48 hours (Fig. 3C). In contrast, AdSPTLC1 infection did not affect cell viability. To
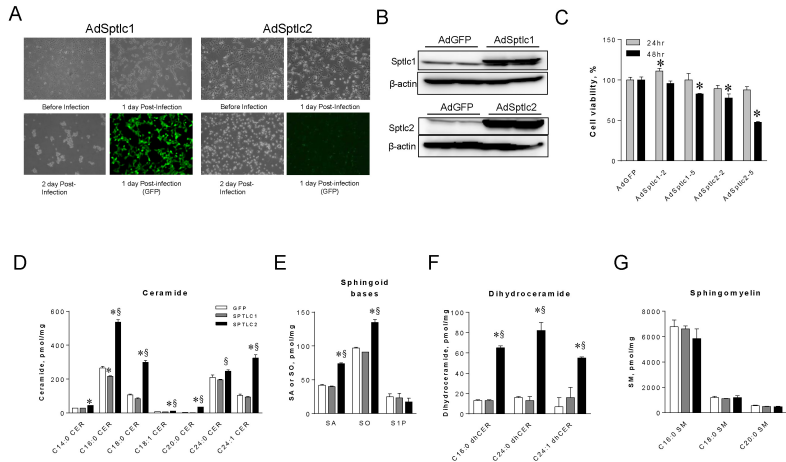

investigate whether SPTLC2 overexpression activates the de novo ceramide biosynthesis pathway, we measured sphingolipid metabolites by LC/MS/MS in HepG2 cells after AdSPTLC1 or AdSPTLC2 infection. Ceramide, sphinganine, sphingosine, and dihydroceramide levels in AdSPTLC2-treated HepG2 cells were higher in cells infected with AdSPTLC2 than in those infected with AdGFP or AdSPTLC1 (Fig. 3D-F). However, sphingosine 1-phosphate and sphingomyelin (SM) levels were not altered by AdSPTLC1 or 2 (Fig. 3E, G). Additionally, inconsistent with the celecoxib results, the levels of long chain and very long chain ceramides increased (Fig. 1). These findings indicate that SPTLC2 expression is cytotoxic due to ceramide accumulation.

\section{Activation of de novo sphingolipid biosynthesis by SPTLC2 overexpression induces apoptosis but not ER stress}

We treated HepG2 hepatoma cells with AdSPTLC viruses to determine if SPTLC2-mediated cytotoxicity is due to ceramidemediated apoptosis. Cell morphology was not altered by AdSPTLC1, but AdSPTLC2 infection caused apoptotic morphology after 24 hours of infection (Fig. 4A). To confirm this, the proportions of apoptotic cells were analyzed by flow cytometry. Apoptosis was significantly increased by AdSPTLC2 infection $(66.1 \%$ of cells in apoptotic phase), compared to
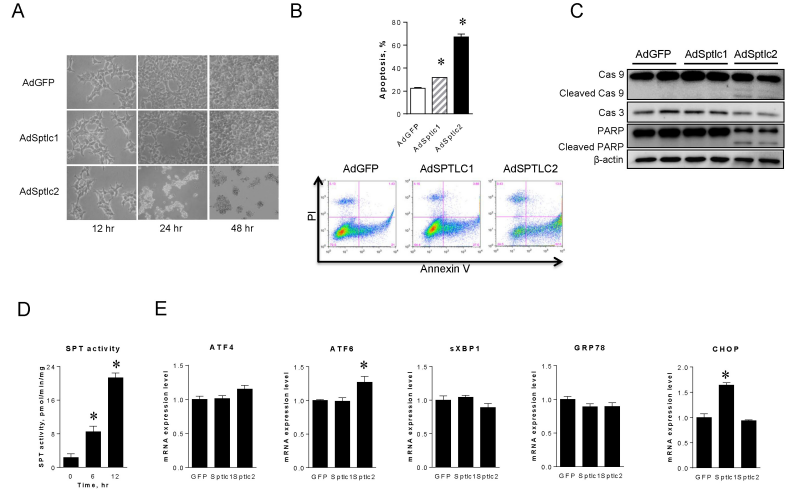

Fig. 3. Adenoviral overexpression of Sptlc2 decreases cell viability in HepG2 cells and elevates cellular sphingolipids. For adenovirus production, HEK293 cells were infected with adenoviruses containing SPTLC1 (AdSPTLC1) and SPTLC2 (AdSPTLC2). Pictures were taken to observe cell morphologies at indicated times post-infection (A). Since GFP is co-expressed with the gene of interest, expression of the gene was observed indirectly with GFP expression. HepG2 cells were infected with adenoviruses containing SPTLC1 or SPTLC2 at $2 \mathrm{MOI}$ (multiplicity of infection) and protein expression was examined by immunoblotting (B). Cell viability was measured by XTT assay after incubation with 2 or 5 $\mathrm{MOI}$ of SPTCL1 and SPTLC2 adenoviruses for 24 and 48 hours (C). Data are presented as the mean \pm SEM. $n=3 * P<0.05$. Sphingolipids were extracted from adenoviral infected HepG2 cells after 24 hours and analyzed by LC/MS/MS. Total ceramides (D), sphinganine (SA), sphingosine (SO), and sphingosine 1-phosphate (S1P) (E), dihydroceramide (dhCer) (F), sphingomyelin (SM) (G) were quantified. Data are presented as the mean \pm SEM. $\mathrm{n}=3 .{ }^{*} \mathrm{P}<0.05$ vs. GFP. ${ }^{\text {S }} \mathrm{P}<0.05$ vs. SPTLC1.

Fig. 4. Adenoviral overexpression of SPTLC2 induces apoptosis in HepG2 cells but does not activate ER stress. HepG2 cells were infected with SPTLC1 or SPTLC2 adenoviruses at $2 \mathrm{MOI}$ for 24 and 48 hours. Cell morphology was changed only after infection of SPTCL2 adenovirus (A). After 24 hours of infection, cells were stained with Annexin $\mathrm{V}$ and propidium iodide and the degree of apoptosis quantified by flow cytometry (B). Data are presented as the mean \pm SEM. $n=3$. $* \mathrm{P}<0.05$. HepG2 cells were infected with adenoviruses at $2 \mathrm{MOI}$ for 24 hours and whole-cell lysates were subjected to immunoblotting analyses of caspase-3 (Cas 3), caspase-9 (Cas 9), and PARP (C). $\beta$-actin was used as a control. Under the same condition, SPT enzyme activity was measured as described in Materials and Methods (D). Then, the expression of ER stress genes including ATF4, ATF6, sXBP1, GRP78, and CHOP was measured by quantitative real-time PCR (E). Amount of mRNA was normalized by $\beta$-actin. Data are presented as the mean \pm SEM. $n=3$. ${ }^{*} P<0.05$. 
$22.43 \%$ or $31.48 \%$ of AdGFP or Ad-SPTLC1 infected cells in the apoptotic phase, respectively (Fig. 4B). Interestingly, the proportion of apoptotic cells was slightly higher after AdSPTLC1 infection than after AdGFP infection. This was probably due to increased SPTLC1 supply to the SPT complex. In addition, cleavage of caspase 9 and PARP, and decreased caspase 3, were only found in the AdSPTLC2 treatment (Fig. $4 C)$. Furthermore, adenoviral overexpression of SPTLC1 or SPTLC2 increased SPT enzyme activity by 3.7- and 9.2-fold, respectively (Fig. 4D). These results indicate that overexpression of SPTLC2, a catalytic subunit of SPT, is implicated in caspase-dependent apoptotic events.

The fact that celecoxib activates ER stress suggests that it may be activated by increased ceramide. To confirm this, we infected cells with adenoviruses and examined transcriptional expression of ER stress markers. However, we did not find any change in ER stress markers including ATF4, ATF6, sXBP1, GRP78, and CHOP (Fig. 4E). SPTLC1 overexpression only upregulated CHOP 1.6 times. These results suggest that activation of de novo sphingolipid biosynthesis, represented by SPTLC2 overexpression, induces apoptosis via ceramide accumulation but not via ER stress.

\section{DISCUSSION}

Sphingolipids are major components of eukaryote cells that play important roles in biological processes. Ceramide is an important metabolic intermediate in the sphingolipid biosynthetic pathway and regulates cell proliferation and apoptosis (19). The elevation of cellular ceramide levels is caused by the activation of the de novo synthetic pathway or the sphingomyelin salvage pathway (20). Based on previous results that celecoxib activates sphingolipid synthesis, our study demonstrated that: 1) celecoxib transcriptionally upregulates the expression of de novo ceramide biosynthetic genes and the sphingomyelinase pathway; 2) activation of the de novo ceramide biosynthetic pathway induces apoptosis via activation of the caspase cascade; and 3) celecoxib activates ER stress in hepatoma cells but ceramide accumulation does not.

Celecoxib is a therapeutic medicine used to manage pain and inflammation in osteoarthritis and rheumatoid arthritis (21). Celecoxib is a selective COX2 inhibitor and nonsteroidal anti-inflammatory drug that inhibits the production of prostaglandins, such as $\mathrm{PGI}_{2}, \mathrm{PGF}_{2 \alpha}, \mathrm{PGD}_{2}$, and $\mathrm{PGE}_{2}$, preventing inflammatory responses in joints and bone $(22,23)$. Previous reports have demonstrated that, among coxib-class drugs, only celecoxib specifically increases the cellular levels of dihydroceramide and/or ceramide in HCT-116 colon cancer cells (24). This result suggests that celecoxib-mediated increased ceramide and dihydroceramide are associated with increased levels of apoptosis. Treatment of HepG2 hepatoma cells with celecoxib upregulated the expression of genes in the de novo ceramide biosynthetic pathway. In addition, sphingomyelinases, which generate ceramide from sphingomyelin, were also upregulated
(Fig. 1A). Interestingly, sphingolipid profiling results showed that long chain ceramides, including C16- and C18-ceramide, were increased by celecoxib. In contrast, the levels of very long chain ceramides, such as C24-ceramide and C24:1ceramide, were not altered. Since long chain ceramides are pro-apoptotic and the very long chain ceramides are associated with cell proliferation, these results suggest that apoptosis is induced by an increased proportion of long chain ceramide species (25-27). These results suggest that alteration of specific ceramide levels and proportion modulates preapoptotic events aroused by celecoxib.

Since both de novo and sphingomyelinase pathways are involved in ceramide production, we first examined whether the de novo pathway contributes to increased apoptosis. When SPT subunits were expressed by adenoviral infection, only the morphology of AdSPTLC2-infected cells was altered, and cell survival decreased in a time-dependent and gene dose-dependent manner. Given that adenoviral SPTLC1 expression had no effect on cell survival, and SPTLC2 expression induced apoptosis, it is likely that SPTLC2 is a major catalytic subunit for SPT reaction.

Consistent with the cell viability results, the flow cytometry analyses demonstrated that cells are entering into the apoptotic stage. Indeed, the proportion of apoptotic cells was significantly increased by adenoviral SPTLC2 expression. SPTLC1 overexpression induced a slightly higher amount of apoptosis than did the GFP controls, indicating that the pairing of SPTLC1 and SPTLC2 subunits was increased by elevated levels of SPTLC1 subunits, as shown previously $(28,29)$. However, endogenous SPTLC2 is limited, resulting in only a slight increase in apoptosis.

The finding that adenoviral expression of SPTLC2 induced cleavage of Caspase-3, -9, and PARP suggests that elevated ceramide causes mitochondrial intrinsic pathway-dependent apoptosis. We found that celecoxib activates the regulatory transcription factors in ER stress in HepG2 hepatoma cells. Expression of XBP1, ATF4, ATF6, and CHOP was gradually upregulated by celecoxib in a time-dependent manner and altered the cell status from normal stage to apoptotic progression. However, adenoviral SPTLC2 expression and activation of de novo ceramide biosynthesis did not affect the expression of transcription factors in ER stress (Fig. 4). These results indicate that elevated endogenous ceramide is not associated with activation of ER stress in these hepatoma cells. Therefore, the involvement of the sphingomyelinase pathway in apoptotic progression deserves further study.

Taken together, our studies identified the roles of de novo ceramide biosynthetic pathway in celecoxib-mediated ceramide increase and apoptosis. This observation is relevant to stressinduced apoptosis based on the sensitivity of cancer cells. This suggests a novel strategy to synergize the anti-cancer activity of celecoxib. Further studies will define proper combination therapies by enhancing the efficacy of anti-cancer treatments with celecoxib, or by modulation of ceramide synthesis in liver cancer. 


\section{MATERIALS AND METHODS}

\section{Cell culture}

HepG2 hepatoma cells were obtained from the American Type Culture Collection (ATCC). The cells were cultured in Dulbecco's modified Eagle medium (DMEM; WelGENE) supplemented with $10 \%$ fetal bovine serum (FBS), $1 \mathrm{U} / \mathrm{ml}$ penicillin, and $1 \mu \mathrm{g} / \mathrm{ml}$ streptomycin and incubated at $37^{\circ} \mathrm{C}$ in a $5 \% \mathrm{CO}_{2}$ atmosphere.

\section{Cell viability}

HepG2 cells seeded on 96-well plates were treated with celecoxib or adenoviruses for 24 and $48 \mathrm{~h}$. Then, cell viability was assessed using the XTT colorimetric solution (WelGENE).

\section{Adenovirus construction}

SPTLC1 and SPTLC2 recombinant adenoviruses (AdSPTLC1, AdSPTLC2) were constructed using an AdEasy Adenoviral Vector System (Stratagene) and a pAdTrack CMV vector as described previously $(30,31)$. As a control, adenovirus expressing green fluorescence protein (AdGFP) was prepared as described above.

\section{Flow cytometric analysis of apoptosis}

HepG2 cells were infected with each adenovirus for $24 \mathrm{~h}$. Cells were double-stained with propidium iodide and Annexin $\mathrm{V}$ according to the manufacturer's instructions (Annexin V-FITC Apoptosis Detection Kit I; BD Biosciences) and analyzed using a FACS Calibur flow cytometer (BD Biosciences) and Flowjo software (Tree Star).

\section{Western blot}

Cells were collected and lysed in lysis buffer $(20 \mathrm{mM}$ Tris- $\mathrm{HCl}$, $\mathrm{pH}$ 7.5, $5 \mathrm{mM}$ EDTA, $100 \mathrm{mM} \mathrm{NaF}, 10 \mathrm{mM} \mathrm{Na} 2 \mathrm{P}_{2} \mathrm{O}_{7}, 2 \mathrm{mM}$ $\mathrm{Na}_{3} \mathrm{VO}_{4}, 1 \%$ Triton $\mathrm{X}-100$, protease inhibitor) by vortexing. Thirty micrograms of protein lysate were subjected to $10 \%$ SDS-polyacrylamide gel electrophoresis followed by immunoblotting analyses (32). Antibodies were used to detect caspase-9, caspase-3, PARP (Cell signaling Technology), XBP-1, ATF4, CHOP (Santa Cruz Biotechnology), ATF6 (Abcam), and $\beta$-actin (Millipore). The blots were detected with enhanced chemiluminescent substrate (Bio-Rad).

\section{RNA extraction and Quantitative Real-Time PCR}

Total RNA was isolated from HepG2 cells using the easy-spin (DNA free) Total RNA Extraction Kit (iNtRON) according to the manufacturer's procedure. Real-Time PCR analysis was performed with StepOnePlus equipment (Applied Biosystems) using SYBR Green Master Mix (Takara). mRNA expression levels were normalized to $\beta$-actin. The primers used were shown previously (33).

\section{Sphingolipid analysis by LC/MS/MS}

HepG2 cells were lysed in lysis buffer for sphingolipid analysis. C17-ceramide was used as an internal standard. One milligram of protein from cell lysates were extracted by $\mathrm{MeOH} / \mathrm{CHCl}_{3}(1: 2, \mathrm{v} / \mathrm{v})$. The organic phase was separated and evaporated under $\mathrm{N}_{2}$. Ceramides (Cer), sphinganine (SA), sphingosine (SO), dihydroceramides (dhCer), and sphingomyelin (SM) were separated by high performance liquid chromatography using a C18 column (XTerra C18, $3.5 \mu \mathrm{m}, 2.1 \times 50$ $\mathrm{mm}$ ) and ionized in positive electrospray ionization (ESI) mode as described by Lee et al. $(33,34)$. [M+]/product ions from corresponding sphingolipid metabolites were monitored for multiple reaction monitoring (MRM) quantification by a tandem mass spectrometer, API 4000 Q-trap (Applied Biosystems, Framingham, MA), interfaced with ESI.

\section{Statistical analysis}

The data are shown as mean \pm SEM. Differences between groups were analyzed by the Student $t$ test. $\mathrm{P}<0.05$ was considered significant.

\section{ACKNOWLEDGEMENTS}

This research was supported by the Bio and Medical Technology Development Program through the National Research Foundation of Korea (NRF), funded by the Korean government (MSIP) (NRF-2014M3A9B6069338) to T.S.P.

\section{CONFLICTS OF INTEREST}

The authors have no conflicting financial interests.

\section{REFERENCES}

1. Kitatani K, Idkowiak-Baldys J and Hannun YA (2008) The sphingolipid salvage pathway in ceramide metabolism and signaling. Cell Signal 20, 1010-1018

2. Menaldino DS, Bushnev A, Sun A et al (2003) Sphingoid bases and de novo ceramide synthesis: enzymes involved, pharmacology and mechanisms of action. Pharmacol Res 47, 373-381

3. Brenner B, Ferlinz K, Grassme H et al (1998) Fas/CD95/ Apo-I activates the acidic sphingomyelinase via caspases. Cell Death Differ 5, 29-37

4. Goldkorn T, Balaban N, Shannon M et al (1998) H2O2 acts on cellular membranes to generate ceramide signaling and initiate apoptosis in tracheobronchial epithelial cells. J Cell Sci 111 (Pt 21), 3209-3220

5. Jenkins GM, Cowart LA, Signorelli P, Pettus BJ, Chalfant CE and Hannun YA (2002) Acute activation of de novo sphingolipid biosynthesis upon heat shock causes an accumulation of ceramide and subsequent dephosphorylation of SR proteins. J Biol Chem 277, 42572-42578

6. Baran Y, Salas A, Senkal CE et al (2007) Alterations of ceramide/sphingosine 1-phosphate rheostat involved in the regulation of resistance to imatinib-induced apoptosis in K562 human chronic myeloid leukemia cells. J Biol Chem 282, 10922-10934 
7. Wang H, Maurer BJ, Reynolds CP and Cabot MC (2001) $\mathrm{N}$-(4-hydroxyphenyl)retinamide elevates ceramide in neuroblastoma cell lines by coordinate activation of serine palmitoyltransferase and ceramide synthase. Cancer Res 61, 5102-5105

8. Perry DK, Carton J, Shah AK, Meredith F, Uhlinger DJ and Hannun YA (2000) Serine palmitoyltransferase regulates de novo ceramide generation during etoposide-induced apoptosis. J Biol Chem 275, 9078-9084

9. Grosch S, Tegeder I, Niederberger E, Brautigam $L$ and Geisslinger G (2001) COX-2 independent induction of cell cycle arrest and apoptosis in colon cancer cells by the selective COX-2 inhibitor celecoxib. FASEB J 15, 2742-2744

10. Kim HS, Kim T, Kim MK, Suh DH, Chung HH and Song YS (2013) Cyclooxygenase-1 and -2: molecular targets for cervical neoplasia. J Cancer Prev 18, 123-134

11. Zhang $\mathrm{H}$, Li Z and Wang $\mathrm{K}$ (2014) Combining sorafenib with celecoxib synergistically inhibits tumor growth of non-small cell lung cancer cells in vitro and in vivo. Oncol Rep 31, 1954-1960

12. Schiffmann S, Sandner J, Schmidt R et al (2009) The selective COX-2 inhibitor celecoxib modulates sphingolipid synthesis. J Lipid Res 50, 32-40

13. Truman JP, Garcia-Barros M, Obeid LM and Hannun YA (2014) Evolving concepts in cancer therapy through targeting sphingolipid metabolism. Biochim Biophys Acta 1841, 1174-1188

14. Schroder M and Kaufman RJ (2005) ER stress and the unfolded protein response. Mutat Res 569, 29-63

15. Puthalakath $\mathrm{H}, \mathrm{O}^{\prime}$ Reilly LA, Gunn $P$ et al (2007) ER stress triggers apoptosis by activating $\mathrm{BH} 3$-only protein Bim. Cell 129, 1337-1349

16. Kucuksayan E, Konuk EK, Demir N, Mutus B and Aslan M (2014) Neutral sphingomyelinase inhibition decreases ER stress-mediated apoptosis and inducible nitric oxide synthase in retinal pigment epithelial cells. Free Radic Biol Med 72, 113-123

17. Senkal CE, Ponnusamy S, Bielawski J, Hannun YA and Ogretmen B (2010) Antiapoptotic roles of ceramidesynthase-6-generated C16-ceramide via selective regulation of the ATF6/CHOP arm of ER-stress-response pathways. FASEB J 24, 296-308

18. Senkal CE, Ponnusamy S, Manevich $Y$ et al (2011) Alteration of ceramide synthase 6/C16-ceramide induces activating transcription factor 6-mediated endoplasmic reticulum (ER) stress and apoptosis via perturbation of cellular $\mathrm{Ca} 2+$ and ER/Golgi membrane network. J Biol Chem 286, 42446-42458

19. Hannun YA and Luberto $C$ (2000) Ceramide in the eukaryotic stress response. Trends Cell Biol 10, 73-80

20. Kolesnick R (2002) The therapeutic potential of modulating the ceramide/sphingomyelin pathway. J Clin Invest
$110,3-8$

21. Hirayama A, Tanahashi N, Daida H et al (2014) Assessing the cardiovascular risk between celecoxib and nonselective nonsteroidal antiinflammatory drugs in patients with rheumatoid arthritis and osteoarthritis. Cir J 78, 194-205

22. Pereira PA, Trindade BC, Secatto A et al (2013) Celecoxib improves host defense through prostaglandin inhibition during Histoplasma capsulatum infection. Mediators Inflamm 2013, 950981

23. Tsuji S, Tomita $\mathrm{T}$, Nakase $\mathrm{T}$, Hamada $\mathrm{M}$, Kawai $\mathrm{H}$ and Yoshikawa H (2014) Celecoxib, a selective cyclooxygenase-2 inhibitor, reduces level of a bone resorption marker in postmenopausal women with rheumatoid arthritis. Int J Rheum Dis 17, 44-49

24. Schiffmann S, Sandner J, Schmidt R et al (2009) The selective COX-2 inhibitor celecoxib modulates sphingolipid synthesis. J Lipid Res 50, 32-40

25. Hla T and Kolesnick R (2014) C16:0-ceramide signals insulin resistance. Cell Metab 20, 703-705

26. Park JW, Park WJ and Futerman AH (2014) Ceramide synthases as potential targets for therapeutic intervention in human diseases. Biochim Biophys Acta 1841, 671-681

27. Liu X, Elojeimy S, Turner LS et al (2008) Acid ceramidase inhibition: a novel target for cancer therapy. Front Biosci 13, 2293-2298

28. Tamehiro N, Zhou S, Okuhira K et al (2008) SPTLC1 binds $A B C A 1$ to negatively regulate trafficking and cholesterol efflux activity of the transporter. Biochemistry 47, 6138-6147

29. Tamehiro N, Mujawar Z, Zhou S et al (2009) Cell polarity factor Par3 binds SPTLC1 and modulates monocyte serine palmitoyltransferase activity and chemotaxis. J Biol Chem 284, 24881-24890

30. Luo J, Deng ZL, Luo X et al (2007) A protocol for rapid generation of recombinant adenoviruses using the AdEasy system. Nat Protoc 2, 1236-1247

31. Chen L, Zou X, Zhang RX et al (2016) IGF1 potentiates BMP9-induced osteogenic differentiation in mesenchymal stem cells through the enhancement of BMP/Smad signaling. BMB Rep 49, 122-127

32. Oh AR, Bae JS, Lee J et al (2016) Ursodeoxycholic acid decreases age-related adiposity and inflammation in mice. BMB Rep 49, 105-110.

33. Lee SY, Hong IK, Kim BR et al (2015) Activation of sphingosine kinase 2 by endoplasmic reticulum stress ameliorates hepatic steatosis and insulin resistance in mice. Hepatology 62, 135-146

34. Yoo HH, Son J and Kim DH (2006) Liquid chromatography-tandem mass spectrometric determination of ceramides and related lipid species in cellular extracts. J Chromatogr B Analyt Technol Biomed Life Sci 843, 327-333 